\title{
Enantioselective Synthesis of (-)-Gilbertine via a Cationic Cascade Cyclization
}

\author{
Jan Jiricek and Siegfried Blechert* \\ Contribution from the Institut für Chemie, Technische Universität Berlin, Strasse des 17. Juni 135, \\ 10623 Berlin, Germany
}

List of supplementary materials

General methods

S2

Experimental procedures

S2

Spectral data

S19 
General methods. All reagents were obtained commercially and were used without further purification, unless indicated in the procedure. Each reaction with air- and moisture- sensitive components was performed under a $\mathrm{N}_{2}$ atmosphere. THF and toluene were distilled from sodium/benzophenone and dichloromethane was distilled from calcium hydride. ${ }^{1} \mathrm{H}$ - and ${ }^{13} \mathrm{C}$ - NMR spectra were recorded at $500 \mathrm{MHz}$ and $125.8 \mathrm{MHz}$ (DRX 500, Bruker). Chemical shifts are expressed in ppm upfield relative to the internal solvent peak. Mass spectra were obtained at an ionizing potential of $70 \mathrm{eV}$ (Finnigan MAT 95 SQ or Varian MAT 711). IR spectra were measured by attenuated total reflection (ATR, Perkin-Elmer spectrometer 881). Elemental analyses were measured with Elementar Vario EI (Analytik Jena). Optical rotations were determined on a polarimeter as solutions in a $10 \mathrm{~cm}$ unit cell at $589 \mathrm{~nm}$ (Perkin-Elmer). GC-MS analyzes were performed with He as carrier gas (GC HP $5890 \mathrm{X})$. The enantiomeric excess was determined by chiral HPLC analyses using DAICEL's CHIRALPAK OD and CHIRALPAK AS columns with hexanes/isopropyl alcohol mixtures as eluent. Melting points are uncorrected.

(S)-2-(3-Oxo-cyclohexyl)-malonic acid dimethyl ester (9). To a solution of R-ALB (1mol\%) was added MS3A (10 g) and the suspension was cooled to $0^{\circ} \mathrm{C}$. KOtBu $(4.3 \mathrm{~mL}, 4.3 \mathrm{mmol})$ was slowly added followed by dimethylmalonate $(28.55 \mathrm{~mL}, 0.25 \mathrm{Mol})$ and cyclohexenone (24.2 mL, 0.25 Mol). The suspension was allowed to warm to room temperature and was stirred for $72 \mathrm{~h}$. The reaction mixture was taken up in EtOAc, filtered over Celite, washed with $1 \mathrm{~N} \mathrm{HCl}$ and dried over sodium sulfate. The solvent was removed in vacuo and the residue was crystallized with hexane/toluene 1:1. Recrystallization afforded 9 (54.77 g, 0.24 Mol, 96\%, >99\%ee, CHIRALPAK AS, eluent: hexanes/2propanol 90:10, flow: $0.5 \mathrm{~mL} / \mathrm{min}$, retention times $[\mathrm{min}]:(\mathbf{R})-9: 39.7,(\mathbf{S})-9: 59.4) .9: \mathrm{mp}: 56^{\circ} \mathrm{C} ;[\alpha]^{20}{ }_{\mathrm{D}}=$ $+3.5\left(\mathrm{c}=0.10, \mathrm{CHCl}_{3}\right) ;{ }^{1} \mathrm{H} \mathrm{NMR}\left(500 \mathrm{MHz}, \mathrm{CDCl}_{3}\right) \delta 1.49(\mathrm{dddd}, J=3,13,13,13 \mathrm{~Hz}, 1 \mathrm{H}), 1.68(\mathrm{ddt}, J$ $=4,13,13 \mathrm{~Hz}, 1 \mathrm{H}), 1.90-1.98(\mathrm{~m}, 1 \mathrm{H}), 2.03-2.12(\mathrm{~m}, 1 \mathrm{H}), 2.20-2.33(\mathrm{~m}, 2 \mathrm{H}), 2.36-2.47(\mathrm{~m}, 2 \mathrm{H}), 2.49-$ $2.60(\mathrm{~m}, 1 \mathrm{H}), 3.34(\mathrm{~d}, J=8 \mathrm{~Hz}, 1 \mathrm{H}), 3.74(\mathrm{~s}, 3 \mathrm{H}), 3.75(\mathrm{~s}, 3 \mathrm{H}) ;{ }^{13} \mathrm{C}$ NMR $\left(500 \mathrm{MHz}, \mathrm{CDCl}_{3}\right) \delta 24.4$ $\left(\mathrm{CH}_{2}\right), 28.7\left(\mathrm{CH}_{2}\right), 38.0(\mathrm{CH}), 40.9\left(\mathrm{CH}_{2}\right), 45.0\left(\mathrm{CH}_{2}\right), 52.5\left(2 \mathrm{CH}_{3}\right), 56.5(\mathrm{CH}), 168.1\left(\mathrm{C}_{\mathrm{q}}\right), 168.2\left(\mathrm{C}_{\mathrm{q}}\right)$, 
$209.4\left(C_{\mathrm{q}}\right)$; IR (ATR) 2954 (w), 2868 (w) 1731 (vs), 1710 (vs), 1434 (s), 1149 (vs); LRMS m/z 228

([M+'], 3), 197 (21), 168 (32), 153 (26), 132 (47), 97 (100); HRMS calcd for $\mathrm{C}_{11} \mathrm{H}_{16} \mathrm{O}_{5}: 228.0997$, found: 228.0996; Anal. calcd for $\mathrm{C}_{11} \mathrm{H}_{16} \mathrm{O}_{5}$ : C, 57.88; H, 7.07, found: C, 58.01; H, 6.81.

(S)-2-(1,4-Dioxa-spiro[4.5]dec-7-yl)-malonic acid dimethyl ester (10). Ketone 9 (52 g, 0.22 Mol) was dissolved in toluene and ethyleneglycol $(38.1 \mathrm{~mL}, 0.68 \mathrm{Mol})$ and para- toluenesulfonic acid (4.3 g, $22.78 \mathrm{mmol}$ ) were added and the resulting mixture was refluxed with a Dean Stark apparatus for $18 \mathrm{~h}$. The reaction mixture was cooled to room temperature and diluted with MTBE, washed several times with water, brine and dried over magnesium sulfate. The solvent was removed in vacuo and the residue was purified by kugelrohr distillation to give $\mathbf{1 0}(61.40 \mathrm{~g}, 0.22 \mathrm{Mol}, 99 \%)$ as a colorless oil. 10: $[\alpha]_{\mathrm{D}}^{20}=$ $+2.8\left(\mathrm{c}=0.14, \mathrm{CHCl}_{3}\right) ;{ }^{1} \mathrm{H} \mathrm{NMR}\left(500 \mathrm{MHz}, \mathrm{CDCl}_{3}\right) \delta 1.07(\mathrm{dddd}, J=3,13,13,13 \mathrm{~Hz}, 1 \mathrm{H}), 1.33(\mathrm{dd}, J$ $=12,13 \mathrm{~Hz}, 1 \mathrm{H}), 1.44(\mathrm{ddd}, J=5,13,13 \mathrm{~Hz}, 1 \mathrm{H}), 1.57(\mathrm{ddt}, J=3,13,13 \mathrm{~Hz}, 1 \mathrm{H}), 1.65-1.82(\mathrm{~m}, 4 \mathrm{H})$, 2.36-2.48 (m, 1H), $3.26(\mathrm{~d}, J=8 \mathrm{~Hz}, 1 \mathrm{H}), 3.72(\mathrm{~s}, 6 \mathrm{H}), 3.90-3.96(\mathrm{~m}, 4 \mathrm{H}) ;{ }^{13} \mathrm{C} \mathrm{NMR}\left(500 \mathrm{MHz}, \mathrm{CDCl}_{3}\right)$ $\delta 22.6\left(\mathrm{CH}_{2}\right), 29.0\left(\mathrm{CH}_{2}\right), 34.6\left(\mathrm{CH}_{2}\right), 35.6(\mathrm{CH}), 38.6\left(\mathrm{CH}_{2}\right), 52.2\left(2 \mathrm{CH}_{3}\right), 56.9\left(\mathrm{CH}_{2}\right), 64.1\left(\mathrm{CH}_{2}\right), 64.2$ $\left(\mathrm{CH}_{2}\right), 108.5\left(\mathrm{C}_{\mathrm{q}}\right), 168.7\left(\mathrm{C}_{\mathrm{q}}\right), 168.8\left(\mathrm{C}_{\mathrm{q}}\right)$; IR (ATR) 2951 (s), 2887 (s), 1754 (vs), 1733 (vs), 1435 (s), 1252 (s), 1191 (w), 1148 (s), 1072 (s); LRMS m/z 272 ([M+], 18), 241 (18), 229 (24), 213 (19), 141 (100), 99 (97). HRMS calcd for $\mathrm{C}_{13} \mathrm{H}_{20} \mathrm{O}_{6}$ : 272.1259, found: 272.1263; Anal. Calcd for $\mathrm{C}_{13} \mathrm{H}_{20} \mathrm{O}_{6}$ : C, 57.34; H, 7.40, found: C, 57.31; H, 7.15.

(S)-2-(1,4-Dioxa-spiro[4.5]dec-7-yl)-acetic acid methyl ester (11). Ketale 10 (61.40 g, $0.22 \mathrm{Mol})$ and DABCO (100 g, 0.89 Mol) were dissolved in toluene and water (16.4 mL, $0.91 \mathrm{Mol})$ was added atroom temperature. The reaction mixture was refluxed for $4 \mathrm{~h}$. The reaction mixture was cooled to room temperature, taken up in MTBE, washed twice with water, brine and dried over magnesium sulfate. The solvent was removed in vacuo and the resulting oil was purified by kugelrohr distillation to give $11(32.21 \mathrm{~g}, 0.15 \mathrm{Mol}, 66 \%)$ as a colorless oil. 11: $[\alpha]^{20}{ }_{\mathrm{D}}=+3.0\left(\mathrm{c}=0.14, \mathrm{CHCl}_{3}\right) ;{ }^{1} \mathrm{H} \mathrm{NMR}(500$ $\left.\mathrm{MHz}, \mathrm{CDCl}_{3}\right) \delta 0.95(\mathrm{dddd}, J=3,13,13,13 \mathrm{~Hz}, 1 \mathrm{H}), 1.23(\mathrm{dd}, J=13 \mathrm{~Hz}), 1.41(\mathrm{ddd}, J=3,13,13 \mathrm{~Hz}$, $1 \mathrm{H}), 1.49-1.61(\mathrm{~m}, 1 \mathrm{H}), 1.68-1.75(\mathrm{~m}, 3 \mathrm{H}), 1.76-1.82(\mathrm{~m}, 1 \mathrm{H}), 2.04-2.16(\mathrm{~m}, 1 \mathrm{H}), 2.23(\mathrm{~d}, J=7 \mathrm{~Hz}$, 
2H), $3.66(\mathrm{~s}, 3 \mathrm{H}), 3.91-3.96(\mathrm{~m}, 4 \mathrm{H}) ;{ }^{13} \mathrm{C} \mathrm{NMR}\left(500 \mathrm{MHz}, \mathrm{CDCl}_{3}\right) \delta 22.8\left(\mathrm{CH}_{2}\right), 31.4\left(\mathrm{CH}_{2}\right), 32.6(\mathrm{CH})$, $34.6\left(\mathrm{CH}_{2}\right), 41.0\left(\mathrm{CH}_{2}\right), 41.1\left(\mathrm{CH}_{2}\right), 51.3\left(\mathrm{CH}_{3}\right), 64.1\left(\mathrm{CH}_{2}\right), 64.2\left(\mathrm{CH}_{2}\right), 108.8\left(\mathrm{C}_{\mathrm{q}}\right), 172.9\left(\mathrm{C}_{\mathrm{q}}\right)$; IR (ATR) 2936 (s), 2883 (w), 1736 (vs), 1436 (w), 1171 (s), 1095 (s), 1068 (s); LRMS m/z 214 ([M+], 9), 183 (8), 171 (57), 141 (58), 99(100); HRMS calcd for $\mathrm{C}_{11} \mathrm{H}_{18} \mathrm{O}_{4}: 214.1205$, found: 214.1210; Anal. calcd for $\mathrm{C}_{11} \mathrm{H}_{18} \mathrm{O}_{4}: \mathrm{C}, 61.66 ; \mathrm{H}, 8.47$, found: $\mathrm{C}, 61.38 ; \mathrm{H}, 8.20$.

(S)-2-(1,4-Dioxa-spiro[4.5]dec-7-yl)-ethanol (12). Lithium aluminiumhydride (6.22 g, $0.15 \mathrm{Mol})$, was suspended in diethyl ether and cooled to $0^{\circ} \mathrm{C}$. The methylester 11 was dissolved in diethylether and was added dropwise to the suspension. After complete addition, the mixture was refluxed for $8 \mathrm{~h}$. The reaction mixture was cooled to room temperature and water was carefully added until a white solid precipitated and hydrogen evolution was completed. The diethylether was decanted and the white solid was washed several times with MTBE. The combined organic layers were washed with water, brine and dried over magnesium sulfate. The solvent was removed in vacuo and the crude oil was purified by kugelrohr destillation to give $12(26.0 \mathrm{~g}, 0.14 \mathrm{Mol}, 97 \%)$ as a colorless oil. 12: $[\alpha]^{20}=+8.6(\mathrm{c}=$ $\left.0.06, \mathrm{CHCl}_{3}\right) ;{ }^{1} \mathrm{H} \mathrm{NMR}\left(500 \mathrm{MHz}, \mathrm{CDCl}_{3}\right) \delta 0.86(\mathrm{dddd}, J=3,13,13,13 \mathrm{~Hz}, 1 \mathrm{H}), 1.18(\mathrm{dd}, J=13,13$ $\mathrm{Hz}, 1 \mathrm{H}), 1.33-1.54(\mathrm{~m}, 4 \mathrm{H}), 1.63-1.78(\mathrm{~m}, 5 \mathrm{H}), 1.99$ (s,br, 1H), 3.59-3.68 (m, 2H), 3.86-3.94 (m, 4H); ${ }^{13} \mathrm{C}$ NMR $\left(500 \mathrm{MHz}, \mathrm{CDCl}_{3}\right) \delta 23.1\left(\mathrm{CH}_{2}\right), 31.8\left(\mathrm{CH}_{2}\right), 32.2(\mathrm{CH}), 34.7\left(\mathrm{CH}_{2}\right), 39.7\left(\mathrm{CH}_{2}\right), 41.7\left(\mathrm{CH}_{2}\right)$, 60.6 ( $\left(\mathrm{CH}_{2}\right), 64.1\left(\mathrm{CH}_{2}\right), 64.3\left(\mathrm{CH}_{2}\right), 109.2\left(\mathrm{C}_{\mathrm{q}}\right)$; IR (ATR $\left.{ }^{-1}\right) 3425$ (w, br), 2930 (vs), $2882(\mathrm{~s}), 1335$ (w), 1074 (vs), 1058 (vs), 1047 (vs); LRMS m/z 186 ([M+'], 6), 143 (68), 141 (49), 99 (100), 86 (28), 55 (12); HRMS calcd for $\mathrm{C}_{10} \mathrm{H}_{18} \mathrm{O}_{3}: 186.1255$, found: 186.1256; Anal. calcd for $\mathrm{C}_{10} \mathrm{H}_{18} \mathrm{O}_{3}:$ C, 64.49; H, 9.74, found: C, 64.52; H, 9.49.

(S)-3-(2-Hydroxy-ethyl)-cyclohexanone (13). Ketal 12 (25.5 g, $0.13 \mathrm{Mol})$ was dissolved in acetonitrile $(500 \mathrm{~mL})$ and $1 \mathrm{~N} \mathrm{HCl}(300 \mathrm{~mL})$ was added at room temperature. The reaction mixture was stirred for $24 \mathrm{~h}$. The reaction mixture was extracted with dichloromethane several times. The combined organic layers were washed with an aqueous saturated sodium hydrogencarbonate solution, brine and dried over magnesium sulfate. The solvent was removed in vacuo and the crude oil was purified by 
column chromatography on silica eluting with hexanes/MTBE (1:6) to yield $\mathbf{1 3}$ (17.71 g, $0.12 \mathrm{Mol}$, 91\%) as a colorless oil. 13: $[\alpha]^{20}=+14.0\left(\mathrm{c}=0.08, \mathrm{CHCl}_{3}\right) ;{ }^{1} \mathrm{H} \mathrm{NMR}\left(500 \mathrm{MHz}, \mathrm{CDCl}_{3}\right) \delta 1.33-1.45(\mathrm{~m}$, 2H), 1.57 (ddt, $J=7,13,13 \mathrm{~Hz}, 1 \mathrm{H}), 1.60-1.73(\mathrm{~m}, 2 \mathrm{H}), 1.89-2.09$ (m, 4H), 2.2-2.3 (m, 1H), 2.32-2.40 (m, 1H), 2.42-2.48 (m, 1H), $3.69(\mathrm{t}, J=7 \mathrm{~Hz}) ;{ }^{13} \mathrm{C} \mathrm{NMR}\left(500 \mathrm{MHz}, \mathrm{CDCl}_{3}\right) \delta 25.1\left(\mathrm{CH}_{2}\right), 31.1\left(\mathrm{CH}_{2}\right)$, $35.6(\mathrm{CH}), 38.9\left(\mathrm{CH}_{2}\right), 41.3\left(\mathrm{CH}_{2}\right), 47.8\left(\mathrm{CH}_{2}\right), 59.7\left(\mathrm{CH}_{2}\right), 212.1\left(\mathrm{C}_{\mathrm{q}}\right)$; IR (ATR) 3408 (w, br), 2927 (s), 2866 (w), 1701 (vs), 1226 (w), 1052 (s), 1040 (s); LRMS m/z 142 ([M+], 9), 124 (7), 99 (51), 97 (100), 81 (20), 67 (21), 55 (42); HRMS calcd for $\mathrm{C}_{8} \mathrm{H}_{14} \mathrm{O}_{2}: 142.0994$, found: 142.0996.

(S)-3-[2-(tert-Butyldiphenylsilanyloxy)-ethyl]-cyclohexanone (14). Ketone 13 (14.22 g, $0.10 \mathrm{Mol})$ and imidazole (13.61 g, 0.20 Mol) were dissolved in DMF (45 mL) and TBDPSCl (29.188 mL, 0.11 Mol, 97\%) was added dropwise at room temperature over a period of $12 \mathrm{~h}$. The reaction mixture was stirred for another $12 \mathrm{~h}$. The reaction mixture was diluted with MTBE washed with water, brine and dried over magnesium sulfate. The solvent was removed in vacuo and the crude oil was purified by column chromatography on silica eluting with hexanes/MTBE (14:1) to give 14 (36.91 g, $0.09 \mathrm{Mol}$, 97\%) as a colorless oil. 14: $[\alpha]^{20}=+9.1\left(\mathrm{c}=0.28, \mathrm{CHCl}_{3}\right) ;{ }^{1} \mathrm{H} \mathrm{NMR}\left(500 \mathrm{MHz}, \mathrm{CDCl}_{3}\right) \delta 1.04(\mathrm{~s}, 9 \mathrm{H})$, $1.23-1.36(\mathrm{~m}, 1 \mathrm{H}), 1.50-1.70(\mathrm{~m}, 3 \mathrm{H}), 1.80-1.90(\mathrm{~m}, 1 \mathrm{H}), 1.95-2.06(\mathrm{~m}, 3 \mathrm{H}), 2.24(\mathrm{ddd}, J=6,13,13 \mathrm{~Hz}$, 1H), 2.30-2.45 (m, 2H), $3.69(\mathrm{t}, J=6 \mathrm{~Hz}, 2 \mathrm{H}), 7.35-7.47(\mathrm{~m}, 6 \mathrm{H}), 7.62-7.68(\mathrm{~m}, 4 \mathrm{H}) ;{ }^{13} \mathrm{C}$ NMR $(500$ $\left.\mathrm{MHz}, \mathrm{CDCl}_{3}\right) \delta 19.1\left(\mathrm{C}_{\mathrm{q}}\right), 25.2\left(\mathrm{CH}_{2}\right), 26.8\left(3 \mathrm{CH}_{3}\right), 31.1\left(\mathrm{CH}_{2}\right), 35.7(\mathrm{CH}), 39.0\left(\mathrm{CH}_{2}\right), 41.4\left(\mathrm{CH}_{2}\right), 48.1$ $\left(\mathrm{CH}_{2}\right), 61.3\left(\mathrm{CH}_{2}\right), 127.6(2 \mathrm{CH}), 127.6(2 \mathrm{CH}), 129.6(2 \mathrm{CH}), 133.7\left(\mathrm{C}_{\mathrm{q}}\right), 133.8\left(\mathrm{C}_{\mathrm{q}}\right), 135.5\left(4 \mathrm{CH}_{2}\right), 211.6$ $\left(\mathrm{C}_{\mathrm{q}}\right)$; IR (ATR $\left.{ }^{-1}\right) 3071$ (w), 2956 (s), 2930 (s), 2857 (s), 1713 (vs), 1428 (s), 1111 (vs), 702 (vs); LRMS m/z 323 ([M+-tert Butyl], 100), 199 (91), 183 (10), 139 (6), 123 (4), 105 (6), 91 (4), 77 (6), 55 (5); HRMS calcd for $\mathrm{C}_{20} \mathrm{H}_{23} \mathrm{O}_{2} \mathrm{Si}\left(\left[\mathrm{M}^{+}\right.\right.$-tert Butyl]): 323.1467 , found: 323.1467 ; Anal. calcd for $\mathrm{C}_{24} \mathrm{H}_{32} \mathrm{O}_{2} \mathrm{Si}$ : C, 75.74; H 8.47, found: C, 75.59; H, 8.26.

\section{(S)-5-[2-(tert-Butyldiphenylsilanyloxy)-ethyl]-2-hydroxymethylene-cyclohexanone (15). Ethyl}

formate (freshly distilled over phosphorus pentoxide, $48.8 \mathrm{~mL}, 0.60 \mathrm{Mol}$ ) was added slowly to a suspension of $\mathrm{NaH}(2.43 \mathrm{~g}, 60.71 \mathrm{mmol}, 60 \%)$ in THF $(20 \mathrm{~mL})$ followed by a dropwise addition of a 
solution of ketone $14(5.77 \mathrm{~g}, 15.18 \mathrm{mmol})$ in THF $(120 \mathrm{~mL})$ at room temperature. After complete addition the reaction mixture was stirred at room temperature for $20 \mathrm{~h}$. Water was added carefully until no hydrogen formation was observed. The reaction mixture was extracted several times with MTBE and the combined organic layers were washed with water, brine and dried over magnesium sulfate. The solvent was removed in vacuo and the crude oil was purified by column chromatography on silica eluting with hexanes/MTBE (14:1) to give $15(4.83 \mathrm{~g}, 11.83 \mathrm{mmol}, 78 \%)$ as a yellow oil. 15: $[\alpha]^{20}=$ $+19.4\left(\mathrm{c}=0.16, \mathrm{CHCl}_{3}\right) ;{ }^{1} \mathrm{H}$ NMR $\left(500 \mathrm{MHz}, \mathrm{CDCl}_{3}\right) \delta 1.08(\mathrm{~s}, 9 \mathrm{H}), 1.19-1.30(\mathrm{~m}, 1 \mathrm{H}), 1.52-1.67(\mathrm{~m}$, 1H), 1.79-1.86 (m, 1H), 1.87-1.97 (m, 1H), $2.02(\mathrm{dd}, J=10,18 \mathrm{~Hz}, 1 \mathrm{H}), 2.26-2.35$ (m, 1H), 2.39 (dt, $J$ =5, $15 \mathrm{~Hz}), 2.44(\mathrm{dd}, J=5,18 \mathrm{~Hz}, 1 \mathrm{H}), 3.75(\mathrm{t}, J=6 \mathrm{~Hz}, 2 \mathrm{H}), 7.35-7.49(\mathrm{~m}, 6 \mathrm{H}), 7.64-7.72(\mathrm{~m}, 4 \mathrm{H})$,

$8.69(\mathrm{~s}, 1 \mathrm{H}) ;{ }^{13} \mathrm{C} \mathrm{NMR}\left(500 \mathrm{MHz}, \mathrm{CDCl}_{3}\right) \delta 19.1\left(\mathrm{C}_{\mathrm{q}}\right), 22.4\left(\mathrm{CH}_{2}\right), 26.8\left(3 \mathrm{CH}_{3}\right), 28.6\left(\mathrm{CH}_{2}\right), 29.4(\mathrm{CH})$, $37.6\left(\mathrm{CH}_{2}\right), 38.3\left(\mathrm{CH}_{2}\right), 61.3\left(\mathrm{CH}_{2}\right), 108.4\left(\mathrm{C}_{\mathrm{q}}\right), 127.6(2 \mathrm{CH}), 129.5(2 \mathrm{CH}), 129.6(2 \mathrm{CH}), 133.7\left(\mathrm{C}_{\mathrm{q}}\right)$, $133.7\left(\mathrm{C}_{\mathrm{q}}\right), 135.5(4 \mathrm{CH}), 184.0\left(\mathrm{C}_{\mathrm{q}}\right), 187.8\left(\mathrm{C}_{\mathrm{q}}\right)$; IR $\left(\mathrm{ATR}^{-1}\right) 3432(\mathrm{w}, \mathrm{br}), 3070(\mathrm{w}), 2953(\mathrm{w}), 2930(\mathrm{~s})$, 2857 (s), 1709 (s), 1589, 1472 (vs), 1111 (vs), 1085 (vs), 701 (vs); LRMS m/z 351([M+-tert Butyl], 29), 323 (7), 273 (100), 199 (74), 183 (12), 139 (14), 123 (6), 91 (6), 77 (9), 57 (6); HRMS calcd for $\mathrm{C}_{21} \mathrm{H}_{23} \mathrm{O}_{3} \mathrm{Si}$ ([M-tert Butyl]): 351.1416, found: 351.1417; Anal. calcd for $\mathrm{C}_{25} \mathrm{H}_{32} \mathrm{O}_{3} \mathrm{Si}$ : C, 73.49; H, 7.89, found: C, 73.43; H, 7.69 .

\section{(2S,3S)-2-Allyl-3-[2-(tert-Butyldiphenylsilanyloxy)-ethyl]-6-hydroxymethylene-cyclohexanone}

(16). Diisopropylamine $(4.19 \mathrm{~mL}, 29.69 \mathrm{mmol})$ was dissolved in THF $(10 \mathrm{~mL})$ and cooled to $0^{\circ} \mathrm{C}$. BuLi (11.36 mL, $28.40 \mathrm{mmol}, 1.6 \mathrm{M}$ in hexane) was added and the mixture was stirred for 20 minutes at $0^{\circ} \mathrm{C}$ and then cooled to $-78^{\circ} \mathrm{C}$. The formylderivative $\mathbf{1 5}(5.27 \mathrm{~g}, 12.91 \mathrm{mmol})$ was dissolved in THF (50 mL) and was added slowly to the cooled solution. After the addition was completed, the resulting mixture was stirred for $4 \mathrm{~h}$ at $-78^{\circ} \mathrm{C}$ followed by addition of allyl bromide $(1.42 \mathrm{~mL}, 16.78 \mathrm{mmol})$. The reaction mixture was allowed to warm up to room temperature overnight and was stirred for additional $20 \mathrm{~h}$. Water was added and the aqueous phase was extracted several times with MTBE. The combined organic layers were washed with brine and dried over magnesium sulfate. The solvent was removed in vacuo 
and the crude oil was purified by column chromatography on silica eluting with hexanes/MTBE (14:1) to give $16(3.94 \mathrm{~g}, 9.65 \mathrm{mmol}, 75 \%)$ as an orange oil. 16: $[\alpha]^{20}=+24.3\left(\mathrm{c}=0.19, \mathrm{CHCl}_{3}\right) ;{ }^{1} \mathrm{H} \mathrm{NMR}(500$ $\left.\mathrm{MHz}, \mathrm{CDCl}_{3}\right) \delta 1.05(\mathrm{~s}, 9 \mathrm{H}), 1.28-1.38(\mathrm{~m}, 1 \mathrm{H}), 1.41-1.50(\mathrm{~m}, 1 \mathrm{H}), 1.67-1.82(\mathrm{~m}, 2 \mathrm{H}), 1.88-1.97(\mathrm{~m}$, 1H), 2.16-2.34 (m, 2H), $2.48(\mathrm{~d}, J=6 \mathrm{~Hz}, 2 \mathrm{H}), 3.65-3.83(\mathrm{~m}, 2 \mathrm{H}), 4.9-5.17(\mathrm{~m}, 2 \mathrm{H}), 5.69-5.80(\mathrm{~m}, 1 \mathrm{H})$, 7.35-7.48 (m, 6H), 7.62-7.75 (m, 4H), $\left.8.74(\mathrm{~s}, 1 \mathrm{H}) ;{ }^{13} \mathrm{C} \mathrm{NMR} \mathrm{(500} \mathrm{MHz,} \mathrm{CDCl}_{3}\right) \delta 19.3\left(\mathrm{C}_{\mathrm{q}}\right), 20.9$ $\left(\mathrm{CH}_{2}\right), 24.8\left(\mathrm{CH}_{2}\right), 27.0\left(3 \mathrm{CH}_{3}\right), 31.4(\mathrm{CH}), 35.3\left(\mathrm{CH}_{2}\right), 35.9\left(\mathrm{CH}_{2}\right), 45.6(\mathrm{CH}), 61.6\left(\mathrm{CH}_{2}\right), 108.7\left(\mathrm{C}_{\mathrm{q}}\right)$, $117.4\left(\mathrm{CH}_{2}\right), 127.7(2 \mathrm{CH}), 127.8(2 \mathrm{CH}), 127.9(\mathrm{CH}), 129.7(2 \mathrm{CH}), 133.8\left(\mathrm{C}_{\mathrm{q}}\right), 133.9\left(\mathrm{C}_{\mathrm{q}}\right), 135.6(\mathrm{CH})$, 135.7 (4CH), $185.1\left(\mathrm{C}_{\mathrm{q}}\right), 189.1(\mathrm{CH})$; IR (ATR) 3437 (w, br), 3071 (w), 2952 (s), 2930 (vs), 2857 (vs), 1709 (s), 1589 (s), 1428 (s), 1111 (vs), 1094 (vs), 701 (vs); LRMS m/z 448([M+1], 1<), 391 (16), 313 (19), 227 (10), 199 (100), 183 (7), 139 (9), 91 (6), 77 (8), 55 (6); HRMS calcd for $\mathrm{C}_{28} \mathrm{H}_{36} \mathrm{O}_{3} \mathrm{Si}: 448.2433$, found: 448.2441; Anal. calcd for $\mathrm{C}_{28} \mathrm{H}_{36} \mathrm{O}_{3} \mathrm{Si}$ : C, 74.95; H, 8.09, found: C, 74.94; H, 7.87.

\section{(2S,3S)-2-Allyl-3-[2-(tert-Butyldiphenylsilanyloxy)-ethyl]-6-(phenyl-hydrazono)-cyclohexanone}

(17). Aniline $(0.90 \mathrm{~mL}, 9.98 \mathrm{mmol})$ was added to a concentrated $\mathrm{HCl}(6 \mathrm{M})$ solution at $0^{\circ} \mathrm{C}$. After stirring 10 minutes, a cold solution of aqueous sodium nitrite $(688 \mathrm{mg}, 9.98 \mathrm{mmol}$ in $10 \mathrm{~mL}$ water) was added slowly at $0^{\circ} \mathrm{C}$ under temperature control. The solution was neutralized with $\mathrm{NaOAc}$, followed by addition of an ice chilled solution of $\mathbf{1 6}(2.985 \mathrm{~g}, 6.65 \mathrm{mmol})$ in THF. The reaction mixture was stirred for $1 \mathrm{~h}$ at $0^{\circ} \mathrm{C}$, then diluted with MTBE and the aqueous layer was extracted twice with MTBE. The combined organic layers were washed with brine and dried over magnesium sulfate. The solvent was removed in vacuo and the crude oil was purified by column chromatography on silica eluting with hexanes/MTBE (16:1) to give $17(2.86 \mathrm{~g}, 5.45 \mathrm{mmol}, 82 \%)$ as an orange oil. 17: $[\alpha]^{20}=+4.6(\mathrm{c}=$ 0.18, $\left.\mathrm{CHCl}_{3}\right) ;{ }^{\mathrm{H}} \mathrm{H} \mathrm{NMR}\left(500 \mathrm{MHz}, \mathrm{CDCl}_{3}\right) \delta 1.07(\mathrm{~s}, 9 \mathrm{H}), 1.40-1.55(\mathrm{~m}, 2 \mathrm{H}), 1.78-1.88(\mathrm{~m}, 1 \mathrm{H}), 1.93-$ $2.08(\mathrm{~m}, 2 \mathrm{H}), 2.23-2.30(\mathrm{~m}, 1 \mathrm{H}), 2.36-2.44(\mathrm{~m}, 1 \mathrm{H}), 2.53-2.62(\mathrm{~m}, 1 \mathrm{H}), 2.63-2.75(\mathrm{~m}, 2 \mathrm{H}), 3.67-3.83$ (m, 2H), 5.06 (dd, $J=16,10 \mathrm{~Hz}, 2 \mathrm{H}), 5.67-5.80(\mathrm{~m}, 1 \mathrm{H}), 7.00(, \mathrm{t}, J=7 \mathrm{~Hz}, 1 \mathrm{H}), 7.25-7.34(\mathrm{~m}, 3 \mathrm{H})$, 7.36-7.49 (m, 6H), 7.64-7.73 (m, 4H); ${ }^{13} \mathrm{C}$ NMR $\left(500 \mathrm{MHz}, \mathrm{CDCl}_{3}\right) \delta 19.1\left(\mathrm{C}_{\mathrm{q}}\right), 26.2\left(\mathrm{CH}_{2}\right), 26.8$ $\left(3 \mathrm{CH}_{3}\right), 29.6\left(\mathrm{CH}_{2}\right), 32.8(\mathrm{CH}), 34.5\left(\mathrm{CH}_{2}\right), 36.4\left(\mathrm{CH}_{2}\right), 53.3(\mathrm{CH}), 61.4\left(\mathrm{CH}_{2}\right), 114.4(\mathrm{CH}), 117.3\left(\mathrm{CH}_{2}\right)$, 
$122.8(\mathrm{CH}), 127.6(\mathrm{CH}), 127.7(\mathrm{CH}), 129.3(\mathrm{CH}), 129.6(\mathrm{CH}), 131.9\left(\mathrm{C}_{\mathrm{q}}\right), 133.73\left(\mathrm{C}_{\mathrm{q}}\right), 133.75\left(\mathrm{C}_{\mathrm{q}}\right)$, $135.3(\mathrm{CH}), 135.5(\mathrm{CH}), 143.1\left(\mathrm{C}_{\mathrm{q}}\right), 198.8\left(\mathrm{C}_{\mathrm{q}}\right)$; IR (ATR) $2955(\mathrm{w}), 2930(\mathrm{~s}), 1639(\mathrm{w}), 1601(\mathrm{~s}), 1515$ (vs), 1428 (s), 1166 (s), 1112 (vs), 701 (vs); LRMS m/z 524 ([M+], 52), 484 (5), 467 (39), 427 (6), 389 (5), 299 (6), 274 (58), 234 (21), 199 (52), 176 (39), 162 (48), 91 (100); HRMS calcd for $\mathrm{C}_{33} \mathrm{H}_{40} \mathrm{~N}_{2} \mathrm{O}_{2} \mathrm{Si}$ : 524.2859, found: 524.2855; Anal. calcd for $\mathrm{C}_{33} \mathrm{H}_{40} \mathrm{~N}_{2} \mathrm{O}_{2} \mathrm{Si}: \mathrm{C}, 75.53 ; \mathrm{H}, 7.68 ; \mathrm{N}, 5.34$ found: C, 75.11; H, $7.63 ; \mathrm{N}, 5.07$.

(S)-2-Allyl-3-(2-hydroxy-ethyl)-2,3,4,9-tetrahydro-carbazol-1-one (18). Hydrazone 17 (200 mg, $0.38 \mathrm{mmol}$ ) was dissolved in trifluoroacetic acid and the reaction mixture was heated to $80^{\circ} \mathrm{C}$ for 1 hour. The solvent was removed in vacuo and the crude oil was purified by column chromatography on silica eluting with hexanes/MTBE (1:5) to give $\mathbf{1 8}(51 \mathrm{mg}, 0.19 \mathrm{mmol}, 50 \%)$ as an pale yellow solid. 18: ${ }^{1} \mathrm{H}$ $\mathrm{NMR}\left(500 \mathrm{MHz}, \mathrm{CDCl}_{3}, \mathrm{MeOH}-\mathrm{D} 4\right)$, diastereomeric ratio 1.5:1, $\delta$ 1.58-1.80 (m, 2H), 2.30-2.70 (m, 1H), $2.80(\mathrm{dd}, J=5,17 \mathrm{~Hz}, 0.6 \mathrm{H}), 2.81(\mathrm{dd}, \mathrm{br}, J=5,17 \mathrm{~Hz}, 0.4 \mathrm{H}), 3,01(\mathrm{dd}, J=5,17 \mathrm{~Hz}, 0.4 \mathrm{H}), 3.18$ $(\mathrm{dd}, J=5,17 \mathrm{~Hz}, 0.6 \mathrm{H}), 3.59-3.69(\mathrm{~m}, 2 \mathrm{H}), 4.93-5.09(\mathrm{~m}, 2 \mathrm{H}), 5.72-5.84(\mathrm{~m}, 1 \mathrm{H}), 7.08(\mathrm{t}, J=7 \mathrm{~Hz}, 1 \mathrm{H})$, 7.27-7.32 (m, 1H), 7.34-7.39 (m, 1H), 7.54-7.60 (m, 1H); $\left.{ }^{13} \mathrm{C} \mathrm{NMR} \mathrm{(500} \mathrm{MHz,} \mathrm{CDCl}_{3}\right)$, diastereomeric ratio 1.5:1, $\delta 23.3\left(\mathrm{CH}_{2}\right), 24.8\left(\mathrm{CH}_{2}\right), 30.1\left(\mathrm{CH}_{2}\right), 33.8\left(\mathrm{CH}_{2}\right), 35.72(\mathrm{CH}), 35.74\left(\mathrm{CH}_{2}\right), 36.9(\mathrm{CH}), 51.2$ $(\mathrm{CH}), 52.0(\mathrm{CH}), 59.9\left(\mathrm{CH}_{2}\right), 60.0\left(\mathrm{CH}_{2}\right), 112.5(\mathrm{CH}), 116.4\left(\mathrm{CH}_{2}\right), 116.9\left(\mathrm{CH}_{2}\right), 120.1(\mathrm{CH}), 121.0$ $(\mathrm{CH}), 121.1(\mathrm{CH}), 125.6\left(\mathrm{C}_{\mathrm{q}}\right), 125.6\left(\mathrm{C}_{\mathrm{q}}\right), 125.8\left(\mathrm{C}_{\mathrm{q}}\right), 126.7\left(\mathrm{C}_{\mathrm{q}}\right), 126.8(\mathrm{CH}), 127.0(\mathrm{CH}), 129.7\left(\mathrm{C}_{\mathrm{q}}\right)$, $130.2\left(\mathrm{C}_{\mathrm{q}}\right), 135.4(\mathrm{CH}), 136.0(\mathrm{CH}), 138.3\left(\mathrm{C}_{\mathrm{q}}\right), 138.5\left(\mathrm{C}_{\mathrm{q}}\right), 193.3\left(\mathrm{C}_{\mathrm{q}}\right)$; IR (ATR) $3275(\mathrm{br}, \mathrm{s}), 2926(\mathrm{br})$, 1641 (vs), 1545 (w), 1474 (s), 1330 (s), 745 (s); LRMS m/z 269 ([M+], 100), 252 (2), 241 (22), 224 (58), 210 (43), 196 (44), 183 (39), 170 (28), 154 (23), 129 (61); HRMS calcd for $\mathrm{C}_{17} \mathrm{H}_{19} \mathrm{NO}_{2}: 269.1415$, found: 269.1415; Anal. calcd for $\mathrm{C}_{17} \mathrm{H}_{19} \mathrm{NO}_{2}: \mathrm{C}, 75.81 ; \mathrm{H}, 7.11 ; \mathrm{N}, 5.20$, found: $\mathrm{C}, 75.21 ; \mathrm{H}, 7.29 ; \mathrm{N}, 4.86$.

\section{Formic acid 2-((S)-2-allyl-1-oxo-2,3,4,9-tetrahydro-1H-carbazol-3-yl)-ethylester (70). Hydrazone}

$17(500 \mathrm{mg}, 0.95 \mathrm{mmol})$ was dissolved in formic acid $(15 \mathrm{ml})$ and the reaction mixture was heated to $80^{\circ} \mathrm{C}$ for 1 hour. The solvent was removed in vacuo and the crude oil was purified by column chromatography on silica eluting with hexanes/MTBE (1:5) to give 19 (184 mg, $0.62 \mathrm{mmol}, 65 \%)$ as an 
pale yellow solid. 19: ${ }^{1} \mathrm{H}$ NMR $\left(500 \mathrm{MHz}, \mathrm{CDCl}_{3}\right)$, diastereomeric ratio 1.5:1, $\delta$ 1.74-1.87 (m, 1H), 1.88-2.04 (m, 1H), 2.35-2.45 (m, 0.4H), 2.49-2.56 (m, 1.2H), 2.56-2.62 (m, 0.8H), 2.63-2.76 (m, 1.3H), 2.76-2.82 (m, 0.4H), $2.86(\mathrm{dd}, J=5,17 \mathrm{~Hz}, 0.6 \mathrm{H}), 2.98(\mathrm{dd}, \mathrm{br}, J=7,17 \mathrm{~Hz}, 0.4 \mathrm{H}), 3.10(\mathrm{dd}, J=5,17$ $\mathrm{Hz}, 0.4 \mathrm{H}), 3.27(\mathrm{dd}, J=5,17 \mathrm{~Hz}, 0.6 \mathrm{H}), 4.20-4.34(\mathrm{~m}, 2 \mathrm{H}), 5.03-5.18(\mathrm{~m}, 2 \mathrm{H}), 5.78-5.95(\mathrm{~m}, 1 \mathrm{H}), 7.16$ $(\mathrm{t}, J=8 \mathrm{~Hz}, 1 \mathrm{H}), 7.35-7.41(\mathrm{~m}, 1 \mathrm{H}), 7.49(\mathrm{~d}, J=8 \mathrm{~Hz}, 1 \mathrm{H}), 7.65(\mathrm{~d}, J=8 \mathrm{~Hz}, 1 \mathrm{H}), 8.05(\mathrm{~s}, 0.6 \mathrm{H}), 8.07$ $(\mathrm{s}, 0.4 \mathrm{H}) ;{ }^{13} \mathrm{C} \mathrm{NMR}\left(500 \mathrm{MHz}, \mathrm{CDCl}_{3}\right)$, diastereomeric ratio 1.5:1, $\delta 23.5\left(\mathrm{CH}_{2}\right), 24.8\left(\mathrm{CH}_{2}\right), 26.9(\mathrm{CH})$, $30.2\left(\mathrm{CH}_{2}\right), 31.9\left(\mathrm{CH}_{2}\right), 33.5\left(\mathrm{CH}_{2}\right), 35.9(\mathrm{CH}), 37.0(\mathrm{CH}), 51.0(\mathrm{CH}), 51.7(\mathrm{CH}), 61.7\left(\mathrm{CH}_{2}\right), 61.9$ $\left(\mathrm{CH}_{2}\right), 112.8(\mathrm{CH}), 116.8\left(\mathrm{CH}_{2}\right), 117.3\left(\mathrm{CH}_{2}\right), 120.5(\mathrm{CH}), 121.1(\mathrm{CH}), 121.2(\mathrm{CH}), 125.8\left(\mathrm{C}_{\mathrm{q}}\right), 125.9$ $\left(\mathrm{C}_{\mathrm{q}}\right), 126.0\left(\mathrm{C}_{\mathrm{q}}\right), 126.4(\mathrm{CH}), 127.0(\mathrm{CH}), 127.1(\mathrm{CH}), 128.63(\mathrm{CH}), 130.0\left(\mathrm{C}_{\mathrm{q}}\right), 135.3(\mathrm{CH}), 135.9(\mathrm{CH})$, $160.9(\mathrm{CH}), 161.0(\mathrm{CH}), 192.1\left(\mathrm{C}_{\mathrm{q}}\right)$; IR (ATR) 3275 (br), 2927 (br), 1723 (vs), 1643 (vs), 1619 (s), 1474 (s), 1330 (s), 1169 (vs), 746 (s); LRMS m/z 297 ([M+], 80), 269 (32), 252 (5), 224 (57), 210 (34), 199 (61), 180 (100), 170 (32), 154 (22), 129 (62); HRMS calcd for $\mathrm{C}_{18} \mathrm{H}_{19} \mathrm{NO}_{3}$ : 297.1365, found: 297.1371.

\section{(S)-2-Allyl-3-[2-(tert-Butyldiphenylsilanyloxy)-ethyl]-2,3,4,9-tetrahydro-carbazol-1-one (20ent).}

The hydrazone $17(2.33 \mathrm{~g}, 4.44 \mathrm{mmol})$ was dissolved in toluene (44 $\mathrm{mL})$ and para-toluenesulfonic acid (1.69 g, $8.88 \mathrm{mmol}$ ) was added. The resulting reaction mixture was refluxed for twenty minutes. The reaction mixture was filtered over a pad of silica with dichloromethane as an eluent. The solvent was removed in vасио and the residue was purified by column chromatography on silica eluting with hexanes/MTBE (4:1) to give 20ent $(1.63 \mathrm{~g}, 3.21 \mathrm{mmol}, 72 \%)$ as a pale yellow foam. 20ent: ${ }^{1} \mathrm{H}$ NMR $\left(500 \mathrm{MHz}, \mathrm{CDCl}_{3}\right)$, diastereomeric ratio 2:1, $\delta$ 1.05-1.15 $(\mathrm{s}, 9 \mathrm{H}), 1.64-1.76(\mathrm{~m}, 1 \mathrm{H}), 1.77-1.96(\mathrm{~m}, 1 \mathrm{H})$, 2.31-2.45 (m, 0.3H), 2.49-2.84 (m, $4.3 \mathrm{H}), 2.86-2.96(\mathrm{~m}, 0.3 \mathrm{H}), 3.04(\mathrm{dd}, J=4,17 \mathrm{~Hz}, 0.3 \mathrm{H}), 3.21$ (dd, $J=5,17 \mathrm{~Hz}, 0.6 \mathrm{H}), 3.73-3.90(\mathrm{~m}, 2 \mathrm{H}), 5.00-5.11(\mathrm{~m}, 0.3 \mathrm{H}), 5.09-5.22(\mathrm{dd}, J=10,17 \mathrm{~Hz}), 5.83-6.0(\mathrm{~m}$, 1H), $7.18(\mathrm{t}, J=8 \mathrm{~Hz}), 7.38-7.48(\mathrm{~m}, 7 \mathrm{H}), 7.51(\mathrm{~d}, J=8 \mathrm{~Hz}, 1 \mathrm{H}), 7.61(\mathrm{~d}, J=8 \mathrm{~Hz}, 1 \mathrm{H}), 7.65-7.79(\mathrm{~m}$, 1H), $9.69(\mathrm{~s}, \mathrm{br}, 0.3 \mathrm{H}), 9.72(\mathrm{~s}, \mathrm{br}, 0.6 \mathrm{H}) ;{ }^{13} \mathrm{C} \mathrm{NMR}\left(500 \mathrm{MHz}, \mathrm{CDCl}_{3}\right)$, diastereomeric ratio 2:1, $\delta$ $19.15\left(\mathrm{C}_{\mathrm{q}}\right), 19.16\left(\mathrm{C}_{\mathrm{q}}\right), 23.6\left(\mathrm{CH}_{2}\right), 25.0\left(\mathrm{CH}_{2}\right), 26.9\left(\mathrm{CH}_{3}\right), 27.0\left(\mathrm{CH}_{3}\right), 30.3\left(\mathrm{CH}_{2}\right), 33.6\left(\mathrm{CH}_{2}\right), 35.7$ $(\mathrm{CH}), 35.8\left(\mathrm{CH}_{2}\right), 37.0(\mathrm{CH}), 51.5(\mathrm{CH}), 52.0(\mathrm{CH}), 61.6\left(\mathrm{CH}_{2}\right), 61.9\left(\mathrm{CH}_{2}\right), 112.72(\mathrm{CH}), 112.74(\mathrm{CH})$, 
116.4 $\left(\mathrm{CH}_{2}\right), 117.0\left(\mathrm{CH}_{2}\right), 120.2(\mathrm{CH}), 121.2(\mathrm{CH}), 121.3(\mathrm{CH}), 126.0\left(\mathrm{C}_{\mathrm{q}}\right), 126.1\left(\mathrm{C}_{\mathrm{q}}\right), 126.6\left(\mathrm{C}_{\mathrm{q}}\right), 126.7$ $(\mathrm{CH}), 126.9(\mathrm{CH}), 127.6(\mathrm{CH}), 127.7(\mathrm{CH}), 129.6(\mathrm{CH}), 130.1\left(\mathrm{C}_{\mathrm{q}}\right), 130.6\left(\mathrm{C}_{\mathrm{q}}\right), 133.6\left(\mathrm{C}_{\mathrm{q}}\right), 133.7\left(\mathrm{C}_{\mathrm{q}}\right)$, $133.8\left(\mathrm{C}_{\mathrm{q}}\right), 135.5(\mathrm{CH}), 135.6(\mathrm{CH}), 135.7(\mathrm{CH}), 136.3(\mathrm{CH}), 138.4\left(\mathrm{C}_{\mathrm{q}}\right), 138.5\left(\mathrm{C}_{\mathrm{q}}\right), 193.0\left(\mathrm{C}_{\mathrm{q}}\right), 193.3$ $\left(\mathrm{C}_{\mathrm{q}}\right)$; IR (ATR $\left.{ }^{-1}\right) 3274$ (br), 2930 (s), 1705 (w), 1642 (vs), 1573 (w), 1545 (w), 1473 (s), 1428 (s), 1330 (s), 1111 (vs), 742 (vs), 701 (vs); LRMS m/z 507 ([M+1, 2), 450 (42), 409 (2), 303 (2), 199 (7), 129 (1); HRMS calcd for $\mathrm{C}_{33} \mathrm{H}_{37} \mathrm{NO}_{2} \mathrm{Si}$ : 507.2594, found: 507.2599; Anal. calcd for $\mathrm{C}_{33} \mathrm{H}_{37} \mathrm{NO}_{2} \mathrm{Si}: \mathrm{C}, 78.06 ; \mathrm{H}$, 7.35; N, 2.76, found: $\mathrm{C}, 77.72 ; \mathrm{H}, 7.36 ; \mathrm{N}, 2.56$.

(rac)-2-Allyl-3-[2-(tert-Butyldiphenylsilanyloxy)-ethyl]-2,3,4,9-tetrahydro-carbazol-1-one (20rac). The hydrazone 17rac (1.55 g, $2.95 \mathrm{mmol})$ was dissolved in toluene $(25 \mathrm{~mL})$ and para- toluenesulfonic acid $(1.24 \mathrm{~g}, 6.49 \mathrm{mmol})$ was added. The resulting reaction mixture was refluxed for twenty minutes. The reaction mixture was filtered over a pad of silica with dichloromethane as an eluent. The solvent was removed in vacuo and the residue was purified by column chromatography on silica eluting with hexanes/MTBE (4:1) to give 20rac (899 $\mathrm{mg}, 1.77 \mathrm{mmol}, 60 \%$ ) as a pale yellow foam. The tetrahydrocarbazole was then dissolved in $\mathrm{MeOH}(10 \mathrm{~mL})$ and $\mathrm{NaOMe}(318 \mathrm{mg}, 5.9 \mathrm{mmol})$ was added and the resulting suspension was left for five days. The reaction mixture was diluted with MTBE and the organic layer was washed with water, brine and dried over magnesium sulfate. The solvent was removed in vacuo and the colorless solid $(899 \mathrm{mg}, 1.77 \mathrm{mmol}$ ) was analyzed with chiral HPLC (CHIRALPAK OD, CHIRACEL, eluent: hexanes/2-propanol 95:5, flow $1 \mathrm{~mL} / \mathrm{min}$, retention times [min]: trans-(S)-20: 10.50, trans-(R)-20: 11.50.). trans-20rac: ${ }^{1} \mathrm{H}$ NMR (500 MHz, $\left.\mathrm{CDCl}_{3}\right) \delta 1.05$ (s, $9 \mathrm{H}), 1.57-1.70(\mathrm{~m}, 1 \mathrm{H}), 1.76-1.87(\mathrm{~m}, 1 \mathrm{H}), 2.43-2.50(\mathrm{~m}, 1 \mathrm{H}), 2.51-2.57(\mathrm{~m}, 1 \mathrm{H}), 2.58-2.68(\mathrm{~m}, 2 \mathrm{H})$, $2.74(\mathrm{dd}, J=5,17 \mathrm{~Hz}, 1 \mathrm{H}), 3.15(\mathrm{dd}, J=5,17 \mathrm{~Hz}, 1 \mathrm{H}), 3.70-3.85(\mathrm{~m}, 2 \mathrm{H}), 5.09$ (dd, $J=17,10 \mathrm{~Hz}), 5.83$ (ddt, 7, 10, $17 \mathrm{~Hz}, 1 \mathrm{H}), 7.15$ (t, $J=8 \mathrm{~Hz}, 1 \mathrm{H}), 7.33-7.47$ (m, 8H), 7.57 (, d, $J=8 \mathrm{~Hz}, 1 \mathrm{H}), 7.62-7.70$ (m, 4H), $8.80(\mathrm{~s}, \mathrm{br}, 1 \mathrm{H}) ;{ }^{13} \mathrm{C} \mathrm{NMR}\left(500 \mathrm{MHz}, \mathrm{CDCl}_{3}\right) \delta 19.2\left(\mathrm{C}_{\mathrm{q}}\right), 23.5\left(\mathrm{CH}_{2}\right), 26.9\left(3 \mathrm{CH}_{3}\right), 33.6\left(\mathrm{CH}_{2}\right)$, $35.7(\mathrm{CH}), 35.9\left(\mathrm{CH}_{2}\right), 52.0(\mathrm{CH}), 61.6\left(\mathrm{CH}_{2}\right), 112.6(\mathrm{CH}), 117.0\left(\mathrm{CH}_{2}\right), 120.3(\mathrm{CH}), 121.3(\mathrm{CH}), 126.1$ $\left(\mathrm{C}_{\mathrm{q}}\right), 126.58\left(\mathrm{C}_{\mathrm{q}}\right), 126.60\left(\mathrm{C}_{\mathrm{q}}\right), 126.9(\mathrm{CH}), 127.64(\mathrm{CH}), 127.66(\mathrm{CH}), 129.6(\mathrm{CH}), 130.1\left(\mathrm{C}_{\mathrm{q}}\right), 133.7$ $\left(\mathrm{C}_{\mathrm{q}}\right), 133.8\left(\mathrm{C}_{\mathrm{q}}\right), 135.5(\mathrm{CH}), 135.6(\mathrm{CH}), 135.7(\mathrm{CH}), 192.9\left(\mathrm{C}_{\mathrm{q}}\right)$. 
carbazol-1-one (21). Indole 20 (2.36 g, $4.65 \mathrm{mmol})$ was dissolved in $20 \mathrm{~mL}$ acetone and $10 \mathrm{~mL}$ water was added, followed by NMO (2.51 g,18.59 mmol) and $\mathrm{OsO}_{4}(118 \mathrm{mg}, 0.46 \mathrm{mmol})$. The reaction mixture was stirred overnight and a saturated aqueous sodium sulfite solution was added. The reaction mixture was diluted with dichloromethane and the aqueous layer was extracted several times with dichloromethane. The combined organic layers were washed with brine and dried over magnesium sulfate. The solvent was removed in vacuo and the crude oil was purified by column chromatography on silica eluting with dichloromethane/MeOH (15:1) to give 21 (2.26 g, 4.18 mmol, 90\%) as a brown foam. 21: ${ }^{1} \mathrm{H}$ NMR $(500 \mathrm{MHz}, \mathrm{MeOH}-\mathrm{D} 4)$, mixture of diastereomers: $\delta$ 0.93-0.97 (s, 9H), 1.48-1.92 (m, 4H), 2.42-2.67 (m, 1.76H), 2.68-2.76 (ddd, $J=4,5,17 \mathrm{~Hz}, 0.7 \mathrm{H}), 2.77-2.96(\mathrm{~m}, 0.9 \mathrm{H}), 3.06(\mathrm{ddd}, J=5,5,17$ $\mathrm{Hz}, 0.6 \mathrm{H}), 3.32-3.55(\mathrm{~m}, 2 \mathrm{H}), 3.60-3.76(\mathrm{~m}, 3 \mathrm{H}), 3.68(\mathrm{t}, J=8 \mathrm{~Hz}, 1 \mathrm{H}), 7.15-7.35$ (m, 8H), 7.137-7.44 $(\mathrm{m}, 1 \mathrm{H}), 7.48-7.59(\mathrm{~m}, 4 \mathrm{H}) ;{ }^{13} \mathrm{C} \mathrm{NMR}\left(500 \mathrm{MHz}, \mathrm{CDCl}_{3}\right)$, mixture of diastereomers: $\delta 19.2\left(\mathrm{C}_{\mathrm{q}}\right), 23.9$ $\left(\mathrm{CH}_{2}\right), 24.6\left(\mathrm{CH}_{2}\right), 26.9\left(\mathrm{CH}_{3}\right), 32.8\left(\mathrm{CH}_{2}\right), 33.0\left(\mathrm{CH}_{2}\right), 36.14\left(\mathrm{CH}_{2}\right), 36.17\left(\mathrm{CH}_{2}\right), 37.8(\mathrm{CH}), 38.3(\mathrm{CH})$, $48.8(\mathrm{CH}), 50.3(\mathrm{CH}), 61.6\left(\mathrm{CH}_{2}\right), 61.7\left(\mathrm{CH}_{2}\right), 66.56\left(\mathrm{CH}_{2}\right), 66.58\left(\mathrm{CH}_{2}\right), 66.7\left(\mathrm{CH}_{2}\right), 69.7(\mathrm{CH}), 71.5$ (CH), $71.7(\mathrm{CH}), 112.56(\mathrm{CH}), 112.6(\mathrm{CH}), 120.42(\mathrm{CH}), 120.45(\mathrm{CH}), 120.48(\mathrm{CH}), 121.41(\mathrm{CH})$, $121.43(\mathrm{CH}), 121.47(\mathrm{CH}), 125.8\left(\mathrm{C}_{\mathrm{q}}\right), 126.0\left(\mathrm{C}_{\mathrm{q}}\right), 127.31(\mathrm{CH}), 127.34(\mathrm{CH}), 127.46(\mathrm{CH}), 127.68$ $(\mathrm{CH}), 128.0\left(\mathrm{C}_{\mathrm{q}}\right), 129.62\left(\mathrm{C}_{\mathrm{q}}\right), 129.66(\mathrm{CH}), 129.71(\mathrm{CH}), 129.9\left(\mathrm{C}_{\mathrm{q}}\right), 133.53\left(\mathrm{C}_{\mathrm{q}}\right), 133.57\left(\mathrm{C}_{\mathrm{q}}\right), 133.60$ $\left(\mathrm{C}_{\mathrm{q}}\right), 133.61\left(\mathrm{C}_{\mathrm{q}}\right), 133.67\left(\mathrm{C}_{\mathrm{q}}\right), 135.51(\mathrm{CH}), 135.53(\mathrm{CH}), 135.55(\mathrm{CH}), 138.47\left(\mathrm{C}_{\mathrm{q}}\right), 138.51\left(\mathrm{C}_{\mathrm{q}}\right), 138.56$ $\left(\mathrm{C}_{\mathrm{q}}\right), 138.65\left(\mathrm{C}_{\mathrm{q}}\right), 194.2\left(\mathrm{C}_{\mathrm{q}}\right), 194.8\left(\mathrm{C}_{\mathrm{q}}\right)$; IR $\left(\mathrm{ATR}^{-1}\right) 3409$ (br), 3291 (br), 2930 (s), $2857(\mathrm{~s}), 1700$ (w), 1640 (vs), 1545 (w), 1472 (s), 1428 (s), 1330 (s), 1111 (vs), 742 (s), 702 (vs); LRMS m/z 541 ([M+], 21), 523 (44), 484 (58), 466 (22), 450 (24), 313 (33), 240 (55), 222 (20), 199 (100), 183 (30), 168 (39); HRMS calcd for $\mathrm{C}_{33} \mathrm{H}_{39} \mathrm{NO}_{4} \mathrm{Si}$ : 541.2648, found: 541.2651; Anal. calcd for $\mathrm{C}_{33} \mathrm{H}_{39} \mathrm{NO}_{4} \mathrm{Si}: \mathrm{C}, 73.16$; H, 7.26; N, 2.59, found: C, 72.69; H, 7.31; N, 2.80.

\section{\{(S)-3-[2-(tert-Butyldiphenylsilanyloxy)-ethyl]-1-oxo-2,3,4,9-tetrahydro-1H-carbazol-2-yl $\}-$}

acetaldehyde (22). The diol $21(2.0 \mathrm{~g}, 3.69 \mathrm{mmol})$ was dissolved in toluene (20 mL) and sodium carbonate $(978 \mathrm{mg}, 9.23 \mathrm{mmol})$ was added followed by $\mathrm{Pb}(\mathrm{OAc})_{4}(2.13 \mathrm{~g}, 4.80 \mathrm{mmol})$. The reaction 
mixture was stirred vigorously for $1 \mathrm{~h}$ and was filtrated over a celite pad. The solvent was removed in vacuo and the residue was taken up in MTBE followed by a filtration over fluorisil $®$ and concentrated in vacuo to give $22(1.79 \mathrm{~g}, 3.51 \mathrm{mmol}, 95 \%)$ as a pale brown foam. 22: ${ }^{1} \mathrm{H}$ NMR (500 $\left.\mathrm{MHz}, \mathrm{CDCl}_{3}\right)$, mixture of diastereomers: $\delta 1.01-1.10(\mathrm{~s}, 9 \mathrm{H}), 1.41-1.51(\mathrm{~m}, 0.6 \mathrm{H}), 1.63-1.69(\mathrm{~m}, 1.6 \mathrm{H}), 1.80-1.90(\mathrm{~m}$, $0.5 \mathrm{H}), 2.34-2.42(\mathrm{dd}, J=5,17 \mathrm{~Hz}, 0.6 \mathrm{H}), 2.42-2.50(\mathrm{~m}, 0.4 \mathrm{H}), 2.62-2.76(\mathrm{~m}, 1.4 \mathrm{H}), 2.89-2.98(\mathrm{~m}, 1 \mathrm{H})$, 3.00-3.13 (m, 1.4H), $3.21(\mathrm{dd}, J=5,17 \mathrm{~Hz}, 0.6 \mathrm{H}), 3.48-3.57(\mathrm{~m}, 0.4 \mathrm{H}), 3.61-3.74(\mathrm{~m}, 1 \mathrm{H}), 3.80-3.89$ (m, 1H), 7.12-7.20 (m, 1H), 7.30-7.47 (m, 8H), 7.50-7.74 (m, 5H), 8.81-8.88 (s, 1H), 9.87-9.95 (s, 1H); ${ }^{13} \mathrm{C}$ NMR $\left(500 \mathrm{MHz}, \mathrm{CDCl}_{3}\right)$, mixture of diastereomers: $\delta 19.13\left(\mathrm{C}_{\mathrm{q}}\right), 25.3\left(\mathrm{CH}_{2}\right), 26.4\left(\mathrm{CH}_{2}\right), 26.9$ $\left(\mathrm{CH}_{3}\right), 30.9\left(\mathrm{C}_{\mathrm{q}}\right), 36.3\left(\mathrm{CH}_{2}\right), 37.8(\mathrm{CH}), 38.6(\mathrm{CH}), 41.0\left(\mathrm{CH}_{2}\right), 41.6\left(\mathrm{CH}_{2}\right), 46.6(\mathrm{CH}), 47.9(\mathrm{CH}), 61.2$ $\left(\mathrm{CH}_{2}\right), 61.8\left(\mathrm{CH}_{2}\right), 112.7(\mathrm{CH}), 120.4(\mathrm{CH}), 121.2(\mathrm{CH}), 121.3(\mathrm{CH}), 125.6\left(\mathrm{C}_{\mathrm{q}}\right), 126.2\left(\mathrm{C}_{\mathrm{q}}\right), 127.0\left(\mathrm{C}_{\mathrm{q}}\right)$, $127.2(\mathrm{CH}), 127.3(\mathrm{CH}), 127.67(\mathrm{CH}), 127.70(\mathrm{CH}), 127.72(\mathrm{CH}), 128.2\left(\mathrm{C}_{\mathrm{q}}\right), 129.69(\mathrm{CH}), 129.72$ $(\mathrm{CH}), 129.75(\mathrm{CH}), 130.1\left(\mathrm{C}_{\mathrm{q}}\right), 130.2\left(\mathrm{C}_{\mathrm{q}}\right), 133.48\left(\mathrm{C}_{\mathrm{q}}\right), 133.53\left(\mathrm{C}_{\mathrm{q}}\right), 133.55\left(\mathrm{C}_{\mathrm{q}}\right), 135.5(\mathrm{CH}), 135.6$ (CH), $138.4\left(\mathrm{C}_{\mathrm{q}}\right), 138.5\left(\mathrm{C}_{\mathrm{q}}\right), 191.1\left(\mathrm{C}_{\mathrm{q}}\right), 200.5(\mathrm{CH}), 200.6(\mathrm{CH})$; IR (ATR $\left.{ }^{-1}\right) 3288$ (br, s), $2930(\mathrm{~s})$, 2856 (s), 1722 (vs), 1640 (vs), 1619 (vs), 1473 (s), 1427 (s), 1332 (s), 1111 (vs), 823 (w), 743 (s), 702 (vs); LRMS m/z 509 ([M+'], 7), 452 (100), 374 (7), 330 (7), 199 (34), 183 (11), 168 (48); HRMS calcd for $\mathrm{C}_{32} \mathrm{H}_{35} \mathrm{NO}_{3} \mathrm{Si}$ : 509.2386, found: 509.2386; Anal. calcd for $\mathrm{C}_{32} \mathrm{H}_{35} \mathrm{NO}_{3} \mathrm{Si}$ : C, 75.40; H, 6.92; N, 2.75, found: $\mathrm{C}, 75.35 ; \mathrm{H}, 7.11 ; \mathrm{N}, 2.83$.

\section{(S)-3-[2-(tert-Butyldiphenylsilanyloxy)-ethyl]-2-[2-(hydroxy-methyl-amino)-ethyl]-2,3,4,9-}

tetrahydro-carbazol-1-one (23). Aldehyde $22(374 \mathrm{mg}, 0.73 \mathrm{mmol})$ was dissolved in isopropanol followed by addition of a solution of $\mathrm{N}$-methylhydroxylamine hydrochloride $(67 \mathrm{mg}, 0.81 \mathrm{mmol})$ in water. The resulting mixture was buffered with an aqueous citric acid/potassiumhydroxide solution to pH 5-6. Sodiumcyanoborhydride (100 mg, $1.59 \mathrm{mmol})$ was added in portions under $\mathrm{pH}$-control. After complete addition the reaction mixture was stirred for $4 \mathrm{~h}$ and was diluted with MTBE. The aqueous layer was extracted four times with MTBE and the combined organic layers were dried over magnesium sulfate. The solvent was removed in vacuo and the crude oil was purified by column chromatography on silica eluting with MTBE to give the hydroxylamine 23 (357 $\mathrm{mg}, 0.66 \mathrm{mmol}, 90 \%$ ) as a colorless foam. 
23: ${ }^{1} \mathrm{H}$ NMR $\left(500 \mathrm{MHz}, \mathrm{CDCl}_{3}\right)$, Diastereomeric ratio 1.5:1, $\delta$ 1.04-1.10 (s, 9H), 1.50-2.1 (m, $\left.3.6 \mathrm{H}\right)$, 2.15-2.33 (m, br, 0.6H), 2.40-2.53 (m, 1H), 2.55-2.92 (m, 7H), 3.05-3.16 (m, 0.6H), 3.66-3.84 (m, 2H), 6.65-6.95 (s, br, 0.8H), 7.05-7.18 (m, 1H), 7.28-7.55 (m, 9H), 7.61-7.74 (m, 4H), 9.6-9.8 (s, br, $1 \mathrm{H}) ;{ }^{13} \mathrm{C}$ $\operatorname{NMR}\left(500 \mathrm{MHz}, \mathrm{CDCl}_{3}\right)$, diastereomeric ratio 1.5:1, $\delta 19.1\left(\mathrm{C}_{\mathrm{q}}\right), 25.4\left(\mathrm{CH}_{2}\right), 26.9\left(\mathrm{CH}_{3}\right), 30.5\left(\mathrm{CH}_{2}\right)$, $36.3\left(\mathrm{CH}_{2}\right), 38.4(\mathrm{CH}), 48.8\left(\mathrm{CH}_{3}\right), 60.6(\mathrm{CH}), 61.3\left(\mathrm{CH}_{2}\right), 61.7\left(\mathrm{CH}_{2}\right), 61.73,112.52(\mathrm{CH}), 112.54$ $(\mathrm{CH}), 116.6\left(\mathrm{C}_{\mathrm{q}}\right), 120.55(\mathrm{CH}), 120.58(\mathrm{CH}), 121.34(\mathrm{CH}), 121.4(\mathrm{CH}), 121.5(\mathrm{CH}), 125.6\left(\mathrm{C}_{\mathrm{q}}\right), 127.3$ $(\mathrm{CH}), 127.4(\mathrm{CH}), 127.7(\mathrm{CH}), 127.73(\mathrm{CH}), 127.75(\mathrm{CH}), 127.76(\mathrm{CH}), 127.78(\mathrm{CH}), 129.77(\mathrm{CH})$, $129.78(\mathrm{CH}), 128.81(\mathrm{CH}), 128.83(\mathrm{CH}), 129.84(\mathrm{CH}), 133.34\left(\mathrm{C}_{\mathrm{q}}\right), 133.39\left(\mathrm{C}_{\mathrm{q}}\right), 133.40\left(\mathrm{C}_{\mathrm{q}}\right), 133.43$ $\left(\mathrm{C}_{\mathrm{q}}\right), 133.53\left(\mathrm{C}_{\mathrm{q}}\right), 135.49(\mathrm{CH}), 135.60(\mathrm{CH}), 135.52(\mathrm{CH}), 135.53(\mathrm{CH}), 135.54(\mathrm{CH}), 138.31\left(\mathrm{C}_{\mathrm{q}}\right)$, $138.37\left(\mathrm{C}_{\mathrm{q}}\right), 138.59\left(\mathrm{C}_{\mathrm{q}}\right), 192.9\left(\mathrm{C}_{\mathrm{q}}\right)$; IR (ATR $\left.{ }^{-1}\right) 3277(\mathrm{br}), 2930(\mathrm{~s}), 2856(\mathrm{~s}), 1704(\mathrm{w}), 1640(\mathrm{vs}), 1620$ (s), 1742 (s), 1428 (s), 1330 (s), 1111 (vs), 741 (vs), 702 (vs); LRMS m/z 540 ([M+], 47), 523 (54), 504 (50), 483 (21), 435 (25), 330 (7), 267 (18), 223 (100), 199 (71), 168 (28), 135 (35); HRMS calcd for $\mathrm{C}_{33} \mathrm{H}_{40} \mathrm{~N}_{2} \mathrm{O}_{3} \mathrm{Si}: 540.2808$, found: 540.2809; Anal. calcd for $\mathrm{C}_{33} \mathrm{H}_{40} \mathrm{~N}_{2} \mathrm{O}_{3} \mathrm{Si}^{*} \mathrm{H}_{2} \mathrm{O}: \mathrm{C}, 70.90 ; \mathrm{H}, 7.68 ; \mathrm{N}, 5.01$, found: C, 70.70; H, 7.54; N, 4.91 .

\section{(S)-3-[2-(tert-Butyldiphenylsilanyloxy)-ethyl]-2-[2-(hydroxy-methyl-amino)-ethyl]-1-methyl-}

2,3,4,9-tetrahydro-1H-carbazol-1-ol (24). The hydroxylamine 23 (300 mg, $0.55 \mathrm{mmol}$ ) was dissolved in THF and the solution was cooled to $-20^{\circ} \mathrm{C}$. MeLi $(1.7 \mathrm{~mL}, 2.77 \mathrm{mmol}, 1.6 \mathrm{M}$ in diethylether) was added and the reaction mixture was allowed to warm to room temperature slowly, stirred for additional $2 \mathrm{~h}$. Water was added and the reaction was diluted with MTBE. The aqueous layer was extracted twice with MTBE and the combined organic layers were dried over magnesium sulfate. The solvent was removed in vacuo and the crude foam was purified by column chromatography on silica eluting with dichloromethane/MeOH (15:1) to give $24(253 \mathrm{mg}, 0.45 \mathrm{mmol}, 82 \%)$ as a colorless foam. 24: ${ }^{1} \mathrm{H}$ NMR $\left(500 \mathrm{MHz}, \mathrm{CDCl}_{3}\right)$, mixture of diastereomers, $\delta 1.07(\mathrm{~s}, 9 \mathrm{H}), 1.24-1.85(\mathrm{~m}, \mathrm{br}, 7 \mathrm{H}), 1.90-2.30(\mathrm{~m}, \mathrm{br}$, 3H), 2.34-3.07 (m, br, 6H), 3.7-4.0 (m, 2H), 7.00-7.20 (m, 2H), 7.22-7.51 (m, 7H), 7.63-7.78 (m, 4H),

8.2-9.0 (s, br, $1 \mathrm{H}) ;{ }^{13} \mathrm{C} \mathrm{NMR}\left(500 \mathrm{MHz}, \mathrm{CDCl}_{3}\right)$, mixture of diastereomers, $\delta 19.16\left(\mathrm{C}_{\mathrm{q}}\right), 19.17\left(\mathrm{C}_{\mathrm{q}}\right)$, 
$24.1(\mathrm{CH}), 26.86\left(\mathrm{CH}_{3}\right), 26.89\left(\mathrm{CH}_{3}\right), 26.91\left(\mathrm{CH}_{3}\right), 26.95\left(\mathrm{CH}_{3}\right), 27.94(\mathrm{CH}), 35.4(\mathrm{CH}), 36.5\left(\mathrm{CH}_{2}\right)$, $49.4\left(\mathrm{CH}_{3}\right), 61.86\left(\mathrm{CH}_{2}\right), 61.87\left(\mathrm{CH}_{2}\right), 72.8\left(\mathrm{CH}_{3}\right), 108.2\left(\mathrm{C}_{\mathrm{q}}\right), 110.90(\mathrm{CH}), 110.92(\mathrm{CH}), 111.0(\mathrm{CH})$, $118.50(\mathrm{CH}), 118.55(\mathrm{CH}), 118.67(\mathrm{CH}), 118.94(\mathrm{CH}), 119.0(\mathrm{CH}), 119.1(\mathrm{CH}), 121.32(\mathrm{CH}), 121.54$ $(\mathrm{CH}), 121.96(\mathrm{CH}), 126.85\left(\mathrm{C}_{\mathrm{q}}\right), 126.92\left(\mathrm{C}_{\mathrm{q}}\right), 127.66(\mathrm{CH}), 127.67(\mathrm{CH}), 129.62(\mathrm{CH}), 129.64(\mathrm{CH})$, $129.65(\mathrm{CH}), 133.74\left(\mathrm{C}_{\mathrm{q}}\right), 133.79\left(\mathrm{C}_{\mathrm{q}}\right), 133.80\left(\mathrm{C}_{\mathrm{q}}\right), 133.83\left(\mathrm{C}_{\mathrm{q}}\right), 133.90\left(\mathrm{C}_{\mathrm{q}}\right), 135.55(\mathrm{CH}), 135.58(\mathrm{CH})$, $136.18\left(\mathrm{C}_{\mathrm{q}}\right), 136.28\left(\mathrm{C}_{\mathrm{q}}\right), 136.34\left(\mathrm{C}_{\mathrm{q}}\right)$; IR (ATR $\left.{ }^{-1}\right) 3321$ (br,s), 2959 (s), 2930 (s), $1704(\mathrm{~s}), 1472$ (s), 1428 (s), 1111 (vs), 1090 (vs), 823 (s), 740 (s), 702 (s); LRMS m/z. 538 ([M+1, 14), 522 (17), 507 (94), 479 (20), 406 (38), 368 (7), 208 (100), 199 (37), 167 (8); HRMS calcd for $\mathrm{C}_{34} \mathrm{H}_{42} \mathrm{~N}_{2} \mathrm{O}_{2} \mathrm{Si}\left[\mathrm{M}^{+}-\mathrm{H}_{2} \mathrm{O}\right]$ : 538.3016, found: 538.3019; Anal. calcd for $\mathrm{C}_{34} \mathrm{H}_{44} \mathrm{~N}_{2} \mathrm{O}_{3} \mathrm{Si}$ : C, 73.34; H, 7.96; N, 5.03 found: C, 72.55; H, $8.18, \mathrm{~N}, 4.83$.

(15(S)-3-[2-(tert-Butyldiphenyl-silanyloxy)-ethyl]-2-[2-(Acetyl-hydroxy-methyl-amino)-ethyl]-1methyl-2,3,4,9-tetrahydro-1H-carbazol-1-ol (25). Tetrahydrocarbazole 24 (200 mg, 3.59 mmol) was dissolved in a mixture of pyridine/acetic anhydride (1:1) at room temperature and was left for eight hours. The solvent was removed in vacuo and the crude foam was purified by column chromatography on silica eluting with MTBE to give 25 (143 mg, $0.23 \mathrm{mmol}, 66 \%$ ) as a colorless foam. Comment: An unpolar product was isolated $\mathbf{2 6}(19 \mathrm{mg}, 0.03 \mathrm{mmol}, 9 \%)$. The overall yield of the acetylation reaction is 75\%. Further transformation of both molecules was done in a parallel manner. 25: ${ }^{1} \mathrm{H}$ NMR $(500 \mathrm{MHz}$, $\left.\mathrm{CDCl}_{3}\right)$, mixture of diastereomers, $\delta 1.06(\mathrm{~s}, 9 \mathrm{H}), 1.30-2.30(\mathrm{~m}, 13 \mathrm{H}), 2.40-3.00(\mathrm{~m}, 6 \mathrm{H}), 3.03-3.17(\mathrm{~m}$, 0.5H), 3.68-3.95 (m, 2H), 7.01-7.20 (m, 2H), 7.27-7.50 (m, 8H), 7.62-7.75 (m, 4H), $7.91(\mathrm{~s}, 0.1 \mathrm{H}), 8.08$ (s, $0.2 \mathrm{H}), 8.18(\mathrm{~s}, 0.25 \mathrm{H}), 8.32(\mathrm{~s}, 0.4 \mathrm{H}) ;{ }^{13} \mathrm{C} \mathrm{NMR}\left(500 \mathrm{MHz}, \mathrm{CDCl}_{3}\right)$, mixture of diastereomers, $\delta .19 .2$ $\left(\mathrm{C}_{\mathrm{q}}\right), 19.5\left(\mathrm{C}_{\mathrm{q}}\right), 19.6\left(\mathrm{CH}_{3}\right), 22.2\left(\mathrm{CH}_{2}\right), 26.88\left(\mathrm{CH}_{3}\right), 26.89\left(\mathrm{CH}_{3}\right), 26.91\left(\mathrm{CH}_{3}\right), 26.94\left(\mathrm{CH}_{3}\right), 27.0\left(\mathrm{C}_{\mathrm{q}}\right)$, $30.6\left(\mathrm{CH}_{2}\right), 34.4(\mathrm{CH}), 35.1\left(\mathrm{C}_{\mathrm{q}}\right), 35.8\left(\mathrm{CH}_{2}\right), 44.6(\mathrm{CH}), 46.8\left(\mathrm{CH}_{3}\right), 59.3\left(\mathrm{CH}_{2}\right), 62.0\left(\mathrm{CH}_{2}\right), 106.7$ $(\mathrm{CH}), 110.7(\mathrm{CH}), 111.1\left(\mathrm{C}_{\mathrm{q}}\right), 114.6(\mathrm{CH}), 119.0(\mathrm{CH}), 119.4(\mathrm{CH}), 119.5\left(\mathrm{C}_{\mathrm{q}}\right), 122.91(\mathrm{CH}), 123.86$ $(\mathrm{CH}), 127.60(\mathrm{CH}), 127.62(\mathrm{CH}), 127.64(\mathrm{CH}), 127.8(\mathrm{CH}), 128.1\left(\mathrm{C}_{\mathrm{q}}\right), 129.5(\mathrm{CH}), 129.6(\mathrm{CH}), 131.2$ $\left(\mathrm{C}_{\mathrm{q}}\right), 133.87\left(\mathrm{C}_{\mathrm{q}}\right), 133.95\left(\mathrm{C}_{\mathrm{q}}\right), 133.96\left(\mathrm{C}_{\mathrm{q}}\right), 135.48(\mathrm{CH}), 135.51(\mathrm{CH}), 135.55(\mathrm{CH}), 135.57(\mathrm{CH})$, $135.59(\mathrm{CH}), 135.60(\mathrm{CH}), 135.61(\mathrm{CH}), 136.9\left(\mathrm{C}_{\mathrm{q}}\right), 137.9\left(\mathrm{C}_{\mathrm{q}}\right), 169.7\left(\mathrm{C}_{\mathrm{q}}\right)$; IR $\left(\mathrm{ATR}^{-1}\right) 3406(\mathrm{br}), 3338$ 
(br), 2959 (s), 2930 (s), 2857 (s), 1755 (s), 1472 (w), 1428 (w), 1226 (s), 1111 (vs), 1093 (vs), 1008 (w),

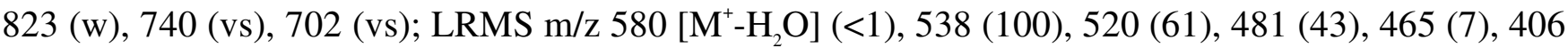
(15), 237 (12), 223 (17), 208 (37), 199 (27), 135 (16); HRMS calcd for $\mathrm{C}_{36} \mathrm{H}_{44} \mathrm{~N}_{2} \mathrm{O}_{3} \mathrm{Si}\left[\mathrm{M}^{+}-\mathrm{H}_{2} \mathrm{O}\right]$ :580.3121, found:580.3129. Unpolar component 26: ${ }^{1} \mathrm{H}$ NMR (500 MHz, MeOH-D4) $\delta 0.96(\mathrm{~s}, 9 \mathrm{H})$, 1.37-1.50 (m, 2H), 1.51-1.65 (m, 2H), 1.80-1.90 (s, 3H), $2.11(\mathrm{~s}, 2.2 \mathrm{H}), 2.14-2.21$ (m, br, 1H), 2.32-2.40 (m, br, 1H), $2.48(\mathrm{~d}, \mathrm{br}, J=16 \mathrm{~Hz}, 1 \mathrm{H}), 2.59(\mathrm{~s}, 3 \mathrm{H}), 2.71-2.90(\mathrm{~m}, 3 \mathrm{H}), 3.60-3.71(\mathrm{~m}, 2 \mathrm{H}), 4.73(\mathrm{~s}, 1 \mathrm{H})$, $5.28(\mathrm{~s}, 1 \mathrm{H}), 6.86(\mathrm{t}, J=7 \mathrm{~Hz}, 1 \mathrm{H}), 7.00(\mathrm{t}, J=7 \mathrm{~Hz}, 1 \mathrm{H}), 7.16-7.40(\mathrm{~m}, 8 \mathrm{H}), 7.52-7.65(\mathrm{~m}, 4 \mathrm{H}) ;{ }^{13} \mathrm{C}$ NMR (500 MHz, MeOH-D4) $\delta 19.6\left(\mathrm{CH}_{3}\right), 20.3\left(\mathrm{C}_{\mathrm{q}}\right), 22.2(\mathrm{CH}), 23.4\left(\mathrm{CH}_{2}\right), 27.8\left(\mathrm{CH}_{3}\right), 32.2\left(\mathrm{CH}_{2}\right)$, $36.3(\mathrm{CH}), 37.4\left(\mathrm{CH}_{2}\right), 45.9\left(\mathrm{CH}_{3}\right), 47.3(\mathrm{CH}), 60.4\left(\mathrm{CH}_{2}\right), 63.5\left(\mathrm{CH}_{2}\right), 108.1\left(\mathrm{CH}_{2}\right), 111.0\left(\mathrm{C}_{\mathrm{q}}\right), 112.1$ $(\mathrm{CH}), 119.8(\mathrm{CH}), 120.0(\mathrm{CH}), 123.6(\mathrm{CH}), 129.1(\mathrm{CH}), 129.4\left(\mathrm{C}_{\mathrm{q}}\right), 131.1(\mathrm{CH}), 133.0\left(\mathrm{C}_{\mathrm{q}}\right), 135.3\left(\mathrm{C}_{\mathrm{q}}\right)$, $135.3\left(\mathrm{C}_{\mathrm{q}}\right), 136.95(\mathrm{CH}), 136.98(\mathrm{CH}), 139.2\left(\mathrm{C}_{\mathrm{q}}\right), 139.83\left(\mathrm{C}_{\mathrm{q}}\right), 168.5\left(\mathrm{C}_{\mathrm{q}}\right), 172.0\left(\mathrm{C}_{\mathrm{q}}\right) ; \mathrm{IR}\left(\mathrm{ATR}^{-1}\right) 3342$ (br,s), 2958 (s), 2930 (s), 1750 (vs), 1704 (w), 1632 (w), 1472 (s), 1428 (vs), 1364 (s), 1232 (br,s), 1111 (vs), 1089 (vs), 823 (s), 737 (vs), 702 (vs); LRMS m/z 580 ([M+] <0.1), 520 (81), 507 (100), 479 (2), 465 (7), 406 (20), 225 (14), 208 (43), 181 (17), 135 (9); HRMS calcd for $\mathrm{C}_{34} \mathrm{H}_{40} \mathrm{~N}_{2} \mathrm{O}_{1} \mathrm{Si}\left[\mathrm{M}^{+}\right.$-HOAc] : 520.2909, found: 520.2904 .

Cyclization precursor (27). The silylether 25 (72 $\mathrm{mg}, 0.120 \mathrm{mmol})$ was dissolved in aqueous THF and TBAF (67 mg, $0.24 \mathrm{mmol}$ ) was added at room temperature. The reaction mixture was stirred for 12 h. After complete reaction the mixture was diluted with MTBE and the aqueous layer was extracted several times with MTBE. The combined organic layers were dried over magnesium sulfate. The solvent was removed in vacuo and the crude foam was purified by column chromatography on silica eluting with dichloromethane/MeOH (20:1) to give 27 (38 $\mathrm{mg}, 0.105 \mathrm{mmol}, 93 \%)$ as a pale yellow foam, which was directly used in the next reaction. A sample $(8 \mathrm{mg}, 0.022 \mathrm{mmol})$ was used for acetylation and conversion to Acetylhydroxyuleine (29) for GC-MS analytics of the cascade reaction.

Gilbertine (2). 27 (30 mg, $0.083 \mathrm{mmol}$ ) was dissolved in trifluoracetic acid and the reaction mixture was left for ten minutes without stirring. The acid was removed in vacuo and the crude foam was 
purified by column chromatography on silica eluting with dichloromethane/methanol/30\%-ammoniasolution $(20: 1: 1)$ to give $2(14 \mathrm{mg}, 0.05 \mathrm{mmol}, 61 \%)$ as a pale green foam. 2 : $[\alpha]^{20}{ }_{\mathrm{D}}=-143.8(\mathrm{c}=$ 0.08, $\left.\mathrm{CHCl}_{3}\right)$; mp: $116-119^{\circ} \mathrm{C} ;{ }^{1} \mathrm{H}$ NMR $\left(500 \mathrm{MHz}, \mathrm{CDCl}_{3}\right) \delta .1 .49(\mathrm{ddd}, J=1.3,3.8,14.1 \mathrm{~Hz}, 1 \mathrm{H}), 1.66$ (s, 3H), 1.80-2.10 (m, 5H), 2.28-2.37 (m, 4H), 2.41-2.48 (m, br, 1H), 2.57-2.66 (m, 1H), $3.42(\mathrm{dd}, J=8$, $12 \mathrm{~Hz}, 1 \mathrm{H}), 4.03(\mathrm{~d}, J=2.6 \mathrm{~Hz}, 1 \mathrm{H}), 7.14(\mathrm{t}, J=8 \mathrm{~Hz}, 1 \mathrm{H}), 7.20(\mathrm{t}, J=8 \mathrm{~Hz}, 1 \mathrm{H}), 7.38(\mathrm{~d}, J=8 \mathrm{~Hz}, 1 \mathrm{H})$, $7.61(\mathrm{~d}, J=8 \mathrm{~Hz}, 1 \mathrm{H}), 8.15(\mathrm{~s}, 1 \mathrm{H}) ;{ }^{13} \mathrm{C} \mathrm{NMR}\left(500 \mathrm{MHz}, \mathrm{CDCl}_{3}\right) \delta .22 .3\left(\mathrm{CH}_{3}\right), 27.0\left(\mathrm{CH}_{2}\right), 29.6\left(\mathrm{CH}_{2}\right)$, $37.8(\mathrm{CH}), 40.7\left(\mathrm{CH}_{3}\right), 44.5(\mathrm{CH}), 45.6\left(\mathrm{CH}_{2}\right), 57.3(\mathrm{CH}), 60.6\left(\mathrm{CH}_{2}\right), 71.0\left(\mathrm{C}_{\mathrm{q}}\right), 108.8\left(\mathrm{C}_{\mathrm{q}}\right), 111.2(\mathrm{CH})$, $119.9(\mathrm{CH}), 120.1(\mathrm{CH}), 122.3(\mathrm{CH}), 127.3\left(\mathrm{C}_{\mathrm{q}}\right), 135.0\left(\mathrm{C}_{\mathrm{q}}\right), 136.2\left(\mathrm{C}_{\mathrm{q}}\right)$; IR ( $\left.\mathrm{ATR}^{-1}\right) 3261(\mathrm{br}, \mathrm{s}), 2969$ (s), 2932 (vs), (2851 (s), 1701 (s), 1451 (vs), 1379 (s), 1324 (s), 1308 (s), 1194 (s), 1084 (s), 1035 (s), 1027 (s), 746 (vs); GC-MS: retention time [min.]: 16.84. m/z 378, 349, 321, 282, 265, 251, 237, 221, 208, 194, 181, 167; LRMS m/z 282 ([M+], 73 ), 267 (9), 251 (10), 239 (14), 237 (17), 225 (20), 208 (8), 194 (20), 181 (20), 168 (17), 110 (26); HRMS calcd for $\mathrm{C}_{18} \mathrm{H}_{22} \mathrm{~N}_{2} \mathrm{O}_{1}: 282.1732$, found:282.1733.

\section{5-Acetylhydroxyuleine (29).}

The sample $(8 \mathrm{mg}, 0.022 \mathrm{mmol})$ was dissolved in acetic anhydride/pyridine 1:1 and the resulting mixture was left for $4 \mathrm{~h}$. The solvent was evaporated and the residue was dissolved in fresh destilled formic acid, resulting in a deep red solution. The solvent was removed and the residue was analyzed via GC-MS. 29: GC-MS: retention time [min]: 18.35. m/z 324, 237, 221, 207, 194, 180, 167, 154; LRMS m/z 324 (8), 269 (1), 237 (7), 207 (11), 194 (6), 180 (3), 167 (3); HRMS calcd for $\mathrm{C}_{20} \mathrm{H}_{24} \mathrm{~N}_{2} \mathrm{O}_{2}$ :324.1837, found:324.1838.

\section{5-Hydroxyuleine (30).}

Tha analytical sample of $\mathbf{2 9}$ was dissolved in $\mathrm{MeOH}$ and an excess of potassium carbonate was added and the resulting suspension was vigorously stirred for $3 \mathrm{~h}$ and filtrated. The solvent was removed in vacuo and the residue was analyzed via GC-MS showing that $\mathbf{3 0}$ decomposes on the column. 30: IR $\left(\mathrm{ATR}^{-1}\right) 3355$ (br, s), 3251 (br, s), 2960 (vs), 2925 (vs), 2854 (s), 1654 (s), 1463 (s), 1260 (s), 1026 (s), 
799 (s), 747 (s); GC-MS: retention times of the decomposed fragments [min.]: 12.86, 13.51, 14.57, 14.82, 15.29, 15.96, 16.32, 17.10; LRMS m/z 282 ([M+'], 8), 269 (8), 237 (8), 231 (9), 208 (10), 194 (10); HRMS calcd for $\mathrm{C}_{18} \mathrm{H}_{22} \mathrm{~N}_{2} \mathrm{O}_{1}: 282.1732$, found: 282.1739 . Comment: Treating 30 with TFA resulted in a $100 \%$ conversion to Gilbertine 2. GC-MS: retention time [min.]: $16.84 . \mathrm{m} / \mathrm{z} 378,349,321,282$, $265,251,237,221,208,194,181,167$.

Ref 14: Calculation of the cis-20 and trans-20 energy differences in the vapour phase.

The starting geometries of the diastereomers were calculated on the basis of MM2 in combination with a Monte Carlo (MC) simulation. Optimization for the minima values was achieved with PM3, AM1 and MNDO until the standard criteria for GAUSSIAN 98 was fulfilled. Furthermore, the PM3 calculation was optimized with BP86 and Ahlrichs TZVP sets which are implemented in TURBOMOLE. (AM1 ($4.53 \mathrm{~kJ} / \mathrm{mol})$, PM3 (-0.15 kJ/mol), MCS (-0.72 kJ/mol) und BP86 (-4.18 kJ/mol)).

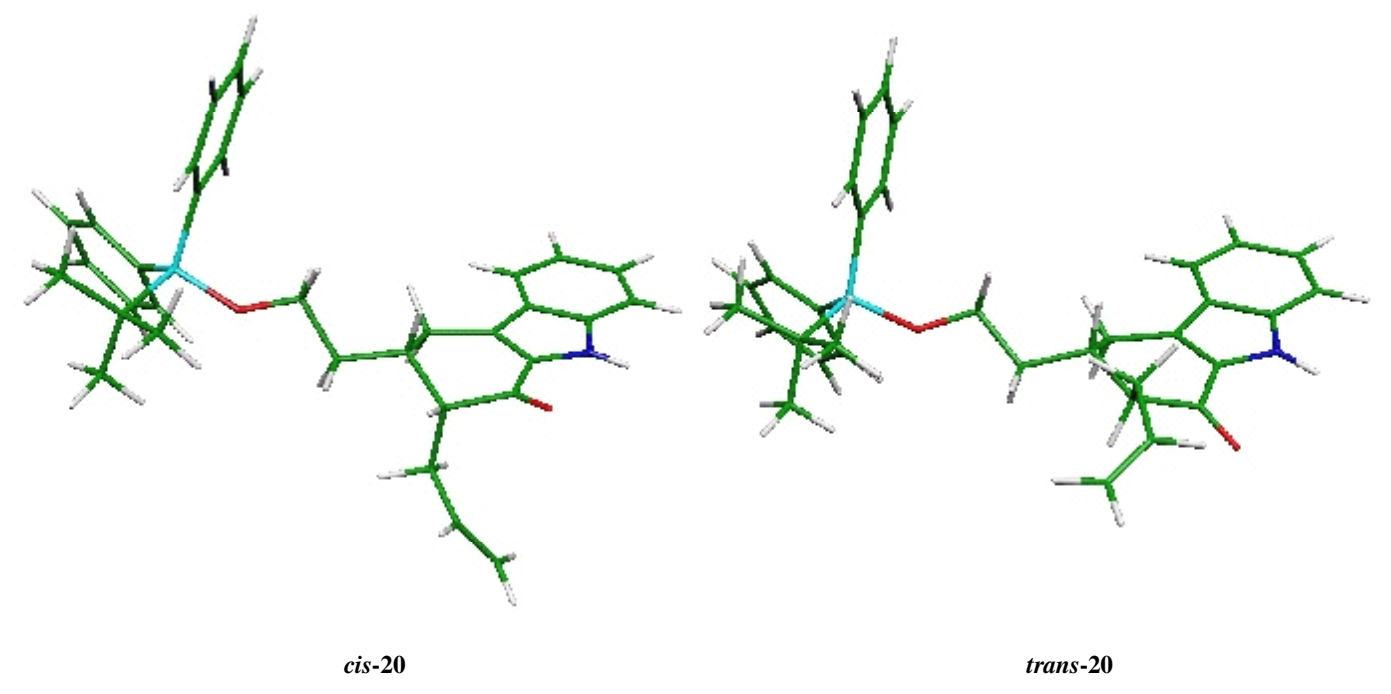




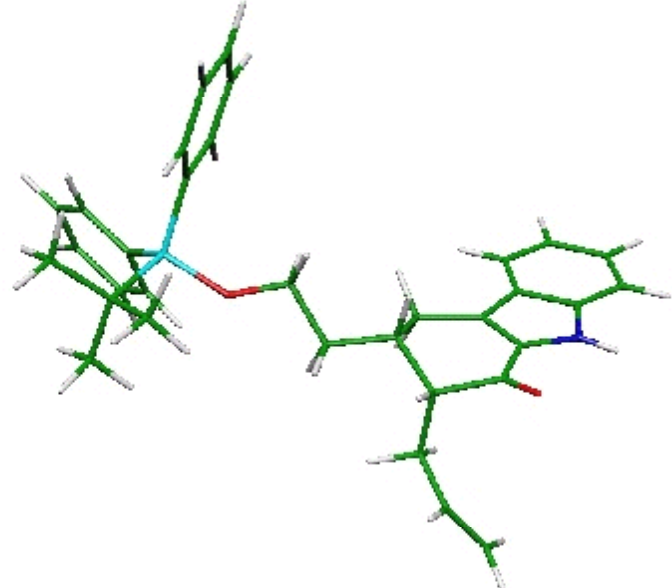

cis-20

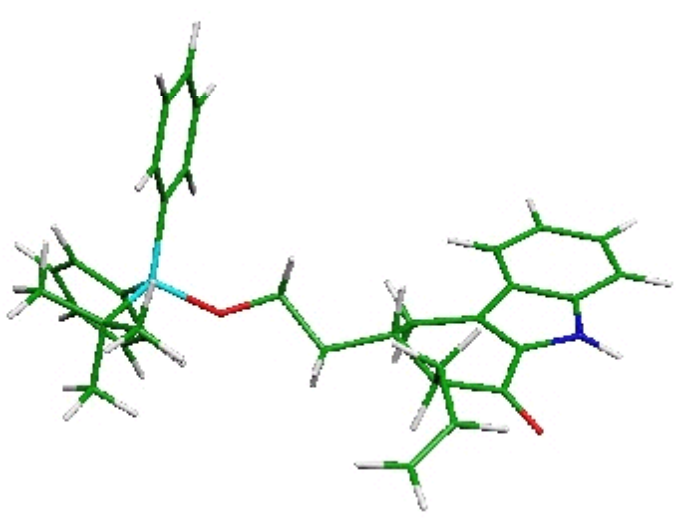

trans-20 